\title{
Robot-assisted frontofacial correction in very young children with craniofacial dysostosis syndromes: a technical note and early functional outcome
}

\author{
*Suhas Udayakumaran, $\mathrm{MCh},{ }^{1}$ Arjun Krishnadas, MDS, ${ }^{2}$ and Pramod Subash, MDS ${ }^{2}$ \\ ${ }^{1}$ Division of Paediatric Neurosurgery and Craniofacial Surgery, Department of Neurosurgery, Amrita Institute of Medical Sciences \\ and Research Centre, Amrita Viswa Vidyapeetham, Kochi, Kerala; and 'Division of Craniomaxillofacial Surgery, Amrita Institute \\ of Medical Sciences and Research Centre, Amrita Viswa Vidyapeetham, Kochi, Kerala, India
}

\begin{abstract}
OBJECTIVE In this study, the authors aimed to 1) retrospectively analyze the early functional outcomes in a cohort of very young children with craniofacial dysostoses who underwent robot-assisted frontofacial advancement (RAFFA) or robot-assisted midface distraction (RAMD), and 2) analyze the utility of robotic assistance in improving the accuracy and safety of performing transfacial pin insertion for RAFFA or RAMD.

METHODS A retrospective analysis of a cohort of 18 children (age range 1-42 months at presentation), who underwent RAFFA or RAMD from February 2015 to February 2021 in the craniofacial unit at Amrita Institute of Medical Sciences and Research Centre in Kochi, India, was performed. Inclusion criteria were patients who had undergone RAFFA in a single stage or RAMD where the cranial vault had been addressed earlier, had been addressed on follow-up, or had not been addressed and had follow-up of at least 6 months.
\end{abstract}

RESULTS Overall, 18 children with syndromic craniosynostosis underwent LeFort level III midface distraction, with or without RAFFA, from February 2015 to February 2021 at a single center in India. The patients' ages ranged from 6 to 47 months at the time of the procedure. All patients had significant obstructive sleep apnea (OSA), significant ocular issues, and disturbed sleep as determined by the authors' preoperative protocol. Clinically significant intracranial pressure issues were present in 17 patients. None of the patients had injury due to the transfacial pin trajectory such as globe injury, damage to the tooth buds, or the loss of purchase during the active distraction phase. The mean distraction achieved was 23 $\mathrm{mm}$ (range 18-30 mm) $(\mathrm{n}=16 / 18)$. Of the 18 patients, $10(56 \%)$ had an excellent outcome and $6(33 \%)$ had a satisfactory outcome. In all cases, the degree of OSA had significantly reduced after surgery. Eye closure improved in all patients, and complete closure was seen in 11 patients. On follow-up, the functional gain remained in 14 of 16 patients at the final follow-up visit. The distraction results were stable during the follow-up period (mean 36 months [range 6-72 months]).

CONCLUSIONS The early RAFFA and RAMD protocols investigated in this study gave a significant functional advantage in very young patients with craniofacial dysostoses. The results have demonstrated the accuracy and safety of robotic assistance in performing transfacial pin insertion for RAFFA or RAMD.

https://thejns.org/doi/abs/10.3171/2021.10.FOCUS21515

KEYWORDS craniosynostosis; midface hypoplasia; faciocraniosynostosis; robotics; obstructive sleep apnea syndrome; functional outcome

$\mathrm{S}$ YNDROMIC craniofacial dysostosis involves the fusion of the cranial sutures and skull base sutures. ${ }^{1-4}$ Clinical features of raised intracranial pressure (ICP) are a constant and early issue in children with this condition. ${ }^{5}$ Additionally, due to midface hypoplasia, these patients have important morphological (e.g., facial retrusion, short nose, ocular dystopia, and strabismus) and functional (breathing difficulties, exorbitism, and recurrent globe dislocation) issues. The resultant, severe obstructive sleep apnea (OSA) can contribute to preexisting raised ICP, which is often unrecognized and underestimated. ${ }^{6-14}$ Moreover, cognitive damage due to OSA without documented raised ICP has also been reported..$^{15-21}$ Most often, raised ICP needs to be addressed, but mid-

ABBREVIATIONS FOAR = fronto-orbital advancement and remodeling; ICP = intracranial pressure; MDCT = multidimensional CT; OSA = obstructive sleep apnea; PROM = patient-reported outcome measure; $\mathrm{PSG}=$ polysomnography; RAFFA = robot-assisted frontofacial advancement; RAMD = robot-assisted midface distraction; STL = stereolithography; URTI = upper respiratory tract infection.

SUBMITTED August 29, 2021. ACCEPTED October 19, 2021.

INCLUDE WHEN CITING DOI: 10.3171/2021.10.FOCUS21515.

* S.U. and P.S. contributed equally to this work. 
face distraction is frequently postponed until the patient is older to avoid relapse., 322

Up to $40 \%$ of patients may require some intervention for OSA..$^{23,24}$ In the presence of severe airway and ocular issues, midface distraction can be performed early. ${ }^{25,26} \mathrm{In}$ this scenario, this may be combined with fronto-orbital advancement and remodeling (FOAR), simultaneously or staged.

We present a series of patients with syndromic craniofacial dysostosis with significant airway compromise, OSA, and ocular crisis, who were treated by robot-assisted midface distraction (RAMD) using transfacial pins $^{27}$ without FOAR or with FOAR (i.e., robot-assisted frontofacial advancement [RAFFA]). There is no dedicated literature similar to our application of robotic assistance in the craniofacial literature other than our previous work on broader applications of robotics in cranial neurosurgery. ${ }^{28}$ Therefore, we aimed to 1) retrospectively analyze the early functional outcome in a cohort of very young children with craniofacial dysostoses who underwent RAFFA and RAMD, and 2) analyze the utility of robotic assistance in improving accuracy and safety while performing transfacial pin insertion for RAFFA and RAMD.

\section{Methods}

The study is a retrospective analysis of a cohort of 18 children, aged 6 to 47 months at the time of the procedure, who underwent RAFFA and/or RAMD from February 2015 to February 2021 in the craniofacial unit at Amrita Institute of Medical Sciences and Research Centre in Kochi, India.

\section{Inclusion Criteria}

Patients were included if they underwent RAFFA in a single stage; underwent RAMD, where the cranial vault had been addressed earlier, had been addressed in the immediate follow-up, or was not addressed; and had a minimum follow-up of 6 months. Figure 1 shows the institutional algorithm for the management of craniofacial dysostosis and the role of RAFFA or RAMD in the protocol. Patient demographics are outlined in Table 1.

\section{Presurgical Planning}

A multidimensional CT (MDCT) scan with a minimum 1-mm slice thickness was obtained within a week of the surgery or on the operating room table using an intraoperative CT scanner.

In the initial stage of the series, before performing the procedure in patients, multiple trials were conducted using the robotic arm-driven drill on a patient-specific 3Dprinted stereolithography (STL) model with a predetermined transfacial transzygomatic trajectory, planned on the ROSA (Zimmer Biomet) planning station, taking care to avoid the tooth buds and the globe (Fig. 2).

\section{Surgical Technique}

The patient is positioned supine on the table, and the head is immobilized using the Sugita head frame (SurgicalOne Inc.), which permits the use of multiple pins, helping to distribute the clamping force and, thus, in turn, stabilizing the head rigidly for registration and accurate transfacial pin insertion using robotic assistance. Where the bone integrity is not adequate for the skull clamp, an on-table, customized, plaster of Paris ring is used to buffer the clamp tip at the skull surface as described in our previous article. ${ }^{29}$

\section{Registration and Planning for Robotic Assistance}

CT scan data of 1-mm slice thickness in DICOM format is loaded onto the ROSA console. The proposed trajectory of the transfacial pin is determined with the help of the software, taking care to obtain the best purchase possible on the hypoplastic zygomatic bone at the entry and exit points while avoiding the orbital contents and developing tooth buds (Figs. 3 and 4). The vector of the proposed pin is assessed in axial, coronal, and sagittal planes and on 3D reconstruction to verify parallelism to the horizontal plane. Surface registration is done to synchronize the CT with the patient. The site is prepped and draped. The robotic arm is then activated to automatically align itself with the patient along the preplanned trajectory. With the help of a drill guide, the transfacial pin $(2.0-\mathrm{mm} \mathrm{K}$ wire) is driven through the bilateral zygomatic bones (Fig. 2). Once the transfacial pin is in position, the Sugita head clamp is removed, and the head is repositioned on a horseshoe clamp for a LeFort III osteotomy and FOAR.

\section{LeFort III Osteotomy and FOAR Surgical Technique}

The procedure for RAMD involves a conventional LeFort III osteotomy, wherein the osteotomy cuts are completed but without down fracture and mobilization, considering the softness of pediatric bone. In RAFFA, FOAR is performed simultaneously to address the craniosynostosis deformity. Care is taken during patient selection to assess the severity of the deformity, and assessment of concomitant raised ICP and the amount of calvarial remodeling is necessary. Therefore, the decision to perform RAFFA versus RAMD was based on the patient's presentation (Fig. 1). Children with calvarial asymmetry or brachycephaly associated with significant expansion in the temporoparietal region that warranted extensive remodeling were not considered for RAFFA, as fixation of the proximal footplate of the distractor device would have been compromised. In these cases, RAMD was performed, followed by subsequent FOAR at the time of the distractor removal.

An isolated RAMD is done using minimally invasive facial incisions placed on the lateral eyebrow and nasal midline, whereas a coronal incision is used for RAFFA. In concomitant LeFort III and FOAR, the osteotomies are placed above and below the frontonasal junction to leave the anterior cranial base intact.

\section{Distractor Device and Fixation}

The distractor is a single linear vector device that consists of a horizontal rod (10-15 mm long, based on the patient's age and head size) that anteriorly rides into a 4-cmlong cylinder with ports for the transfacial pin, which posteriorly engages a connector for the K-wires that fix 


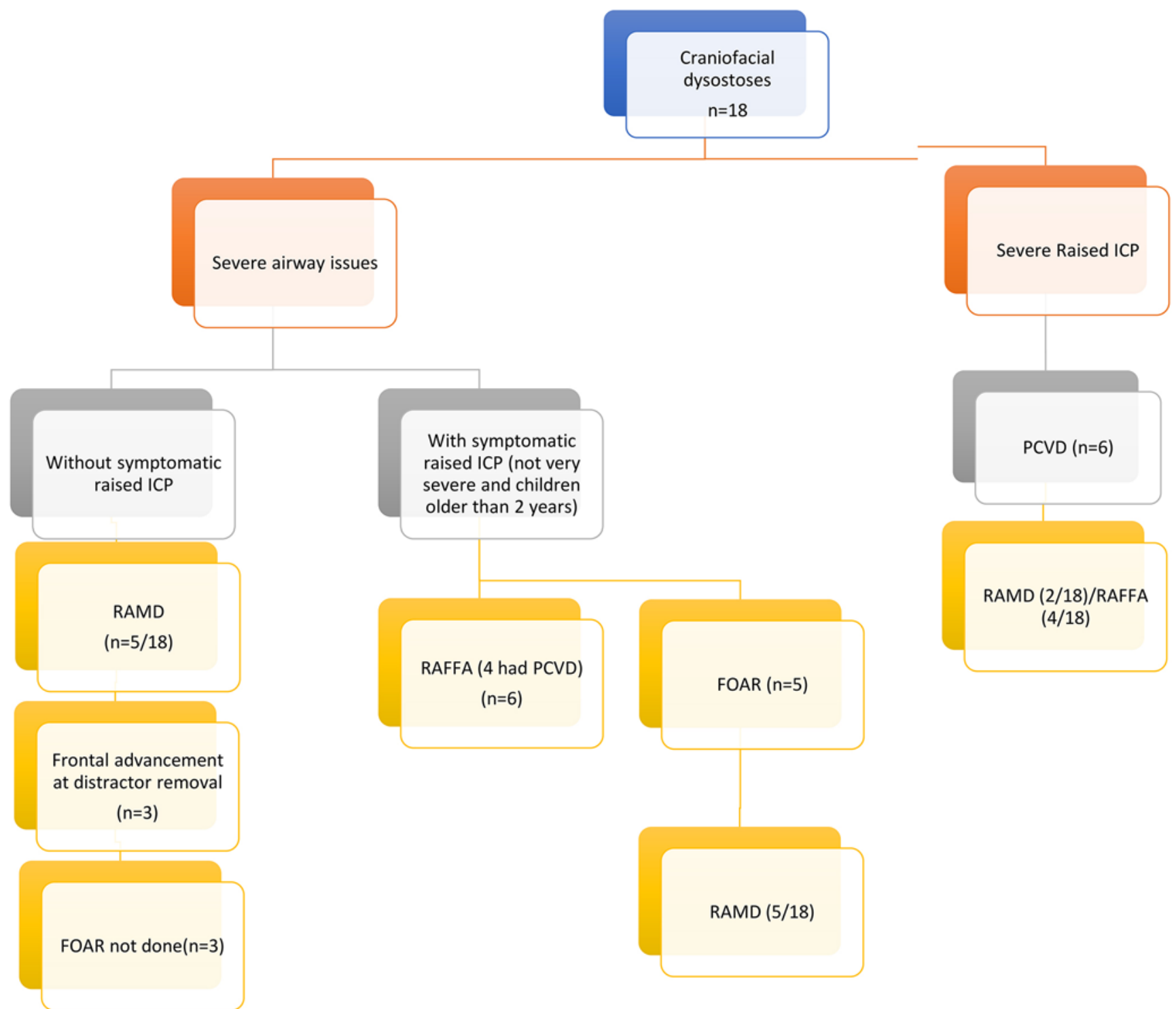

FIG. 1. Institutional algorithm with inclusion data for the management of patients with craniofacial dysostoses and the role of RAFFA or RAMD in the protocol.

the footplate on the cranium (Figs. 4 and 5). The posterior footplate of the distractor consists of a hook that engages the bone through a small burr hole in the temporal bone, a three-hole vertical plate that is fixed to the bone using 1.7-mm self-tapping screws for additional stability, and a U-shaped 1.8-mm K-wire that is passed through the footplate, which engages the connector on the distractor (Fig. 5 and $5 \mathrm{~A}$ inset). A pair of distractors is placed bilaterally to engage the transfacial pin on both sides.

\section{Distraction Protocol and Consolidation}

Distraction was initiated from the 1st postoperative day at the rate of 1 to $2 \mathrm{~mm}$ per day in 2 increments. On the operating table, an early accelerated distraction was performed in selected patients who had severe airway issues. Serial skull radiographs were obtained to assess the progress and vector of the distraction as well as device integrity. The endpoint of distraction was assessed by functional improvement that included reduced snoring, disappearance of chest indrawing, reduced use of the accessory muscles of inhalation, improved sleep patterns, and improved eye closure. The distractor was left in place for a minimum of 3 months for consolidation (Fig. 4). CT scanning was repeated before the following procedure (Fig. 4). Postplacement confirmation CT scans were avoided to reduce cumulative radiation exposure.

Our institutional protocol for assessing the significance of midface retrusion in craniofacial syndromes include the following: 1) recurrent upper respiratory tract infection (URTI) and/or pneumonia, and hospital admission with or without features of failure to thrive; 2) poor sleep quality with respiratory distress and frequent waking; 3) 
TABLE 1. Demographics of patients who underwent RAFFA and RAMD

\begin{tabular}{|c|c|c|c|c|c|c|}
\hline $\begin{array}{l}\text { Pt } \\
\text { No. }\end{array}$ & $\begin{array}{c}\text { Age at 1st } \\
\text { Procedure (mos) }\end{array}$ & $\begin{array}{l}\text { Age at RAFFA/ } \\
\text { RAMD (mos) }\end{array}$ & $\begin{array}{l}\text { Syndromic } \\
\text { Diagnosis }\end{array}$ & $\begin{array}{l}\text { Deciding } \\
\text { Presentation }\end{array}$ & Procedure & $\begin{array}{l}\text { Other Procedures } \\
\text { (in order) }\end{array}$ \\
\hline 1 & 6 & 12 & Apert's & Airway & RAMD & FOAR \\
\hline 2 & 10 & 32 & Apert's & Airway & RAMD & FOAR \\
\hline 3 & 14 & 16 & Apert's & ICP, airway & RAMD & FOAR \\
\hline 4 & 14 & 20 & Apert's & Airway & RAFFA, RAMD & Syndactyly release \\
\hline 5 & 1 & 47 & Crouzon & ICP, airway & RAFFA & ETV \\
\hline 6 & 22 & 22 & Crouzon & ICP, airway & RAFFA & None \\
\hline 7 & 31 & 31 & Crouzon & Airway & RAMD & Tracheostomy \\
\hline 8 & 10 & 43 & Crouzon & ICP, airway & RAFFA & PCVD \\
\hline 9 & 9 & 13 & Crouzon & ICP, airway, globe subluxation & RAFFA & PCVD \\
\hline 10 & 9 & 14 & Crouzon & ICP, airway & RAFFA & PCVD \\
\hline 11 & 4 & 8 & Antley-Bixler & ICP, airway & RAMD & ETV, VP shunt, PCVD, FOAR \\
\hline 12 & 6 & 14 & Crouzon & ICP, airway & RAMD & PCVD, VP shunt \\
\hline 13 & 19 & 19 & Crouzon & Globe subluxation, airway & RAMD & VP shunt \\
\hline 14 & 16 & 16 & Apert's & ICP, airway & RAMD & FOAR \\
\hline 15 & 14 & 17 & Crouzon & ICP, globe subluxation, airway & RAMD & PCVD \\
\hline 16 & 7 & 13 & Crouzon & ICP, airway & RAMD & PCVD \\
\hline 17 & 9 & 10 & Crouzon & ICP, airway & RAMD & PCVD, FOAR, VP shunt \\
\hline 18 & 42 & 42 & Apert's & Airway & RAMD & $\begin{array}{c}\text { None until last FU; pt awaiting FOAR } \\
\text { \& syndactyly release }\end{array}$ \\
\hline
\end{tabular}

ETV = endoscopic third ventriculostomy; FU = follow-up; PCVD = posterior calvarial vault distraction; $p t=$ patient; $V P$ = ventriculoperitoneal .

polysomnography (PSG) with obstructive findings (this is not a strict inclusion criterion when abnormal functional status has been convincing; a screening PSG is acceptable when a complete study is not feasible); and 4) significant exorbitism with incomplete eyelid closure (exposure keratitis, corneal opacity, globe subluxation, or imminent globe subluxation).

\section{Follow-Up and Early Outcome Evaluation}

The follow-up period for this study was 6 months: 3 months to discern the response of the technique (distraction and consolidation phase) and 3 months to evaluate the immediate clinical response. The outcome evaluation of the technique and early functional benefit were based on 1) the ability to pass the transfacial pin in a single attempt using robotic guidance; 2) the ability to maintain parallelism of the pin to the Frankfort horizontal plane for bilaterally symmetrical vectors; 3 ) successful completion of the distraction without transfacial pin dislodgment; and 4) the patient-reported outcome measure (PROM) at the 6-month follow-up (Supplemental Table 1). The outcome analysis was based on the PROM scale in comparison with the preoperative symptoms. The radiological evaluation of the distraction was based on a plain lateral radiograph or MDCT scan, if available.

\section{Statistical Analysis}

Statistical analyses were performed using Microsoft Office Excel. Continuous variables are expressed as the mean \pm SD. All analyses were performed using IBM SPSS Statistics version 20.0 (IBM Corp.).

\section{Results}

After institutional board clearance, 18 children with syndromic craniosynostosis (18/47) underwent LeFort level III midface distraction, with or without FOAR, with robotic assistance from February 2015 to February 2021 in the craniofacial unit of Amrita Institute of Medical Sciences and Research Centre in Kochi, India.

Patients who underwent the procedure ranged in age from 6 to 47 months (age range at presentation 1-42 months). The overall mean age at the time of the surgical procedure (RAFFA and RAMD) was 19.6 months, while the mean age for patients who underwent RAFFA was 26 months (range 13-47 months), and the mean age for those who underwent RAMD was 19 months (range 8-42 months).

All 18 patients had significant OSA, globe issues, and disturbed sleep, as determined by our preoperative protocol (see Methods). Failure to thrive was a significant issue in 10 patients and clinically significant ICP issues were present in 17 patients. Although PSG is part of our algorithm for syndromic craniofacial dysostosis, for the entire cohort, the clinical presentation was so apparent that we did not have to rely on a sleep study for decision-making; nonetheless, 6 patients had PSG with significant obstructive features.

Six patients (6/18) underwent RAFFA in a single stage and 8 patients $(8 / 18)$ underwent the procedure in 2 stages (i.e., RAMD followed by frontal advancement [3/6] or FOAR followed by RAMD [5/18]). Three patients underwent midface distraction alone, as previously described in the algorithm (Fig. 1).

The mean estimated blood loss was $100 \pm 50 \mathrm{ml}$ for 

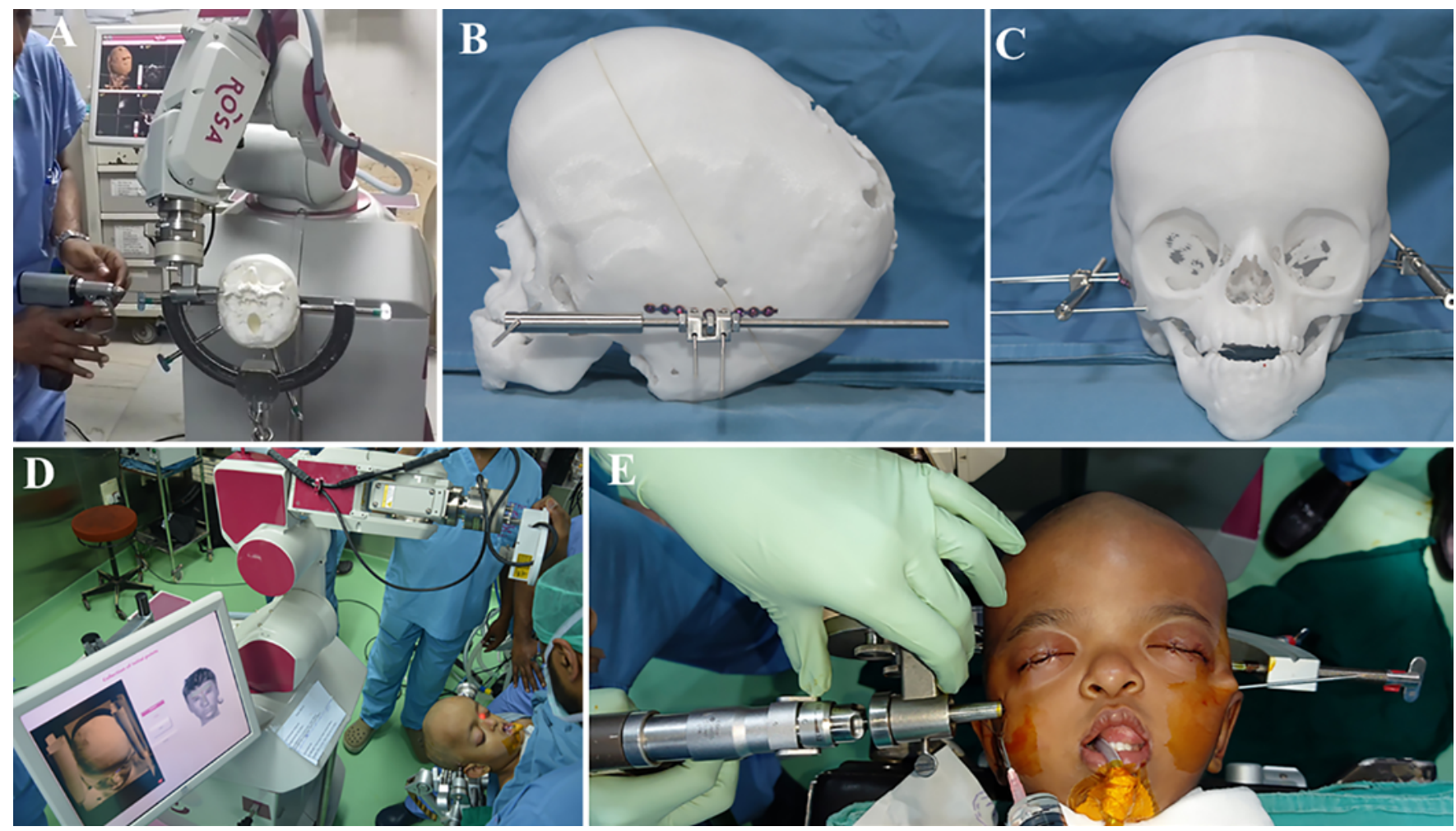

FIG. 2. Setup and technique. A: Planning of robot-assisted transfacial pin insertion. In the initial stage of the series, before performing the procedure in patients, multiple trials were conducted using the robotic arm-driven drill on a patient-specific, 3D-printed STL model with a predetermined transfacial transzygomatic trajectory planned on the ROSA planning station, with consideration to avoid the tooth buds and the globe. B and C: The STL model with the distractor in situ. D: The setup comprising the robotic arm before automated placement into a position for pin insertion, with the patient's head fixed on the Sugita head clamp. E: MDCT scan data of 1-mm slice thickness in DICOM format is loaded onto the ROSA console. Surface registration is done to synchronize the CT with the patient. The site is prepped. The robotic arm automatically aligns with the patient along the preplanned trajectory. With the help of a drill guide, a transfacial pin (2-mm K-wire) is passed through the bilateral zygomatic bones.

RAMD and $450 \pm 25 \mathrm{ml}$ for RAFFA. The mean total duration of the procedure for RAMD was $200 \pm 25$ minutes and for RAFFA, $320 \pm 40$ minutes. Of this mean duration, approximately $60 \pm 20$ minutes was for trajectory planning, registration, and introducing the transfacial pin with robotic assistance. In all 18 patients, the transfacial pin could be introduced in a single passage. In 1 patient, the robotic procedure was abandoned for the conventional procedure due to registration failure (and, hence, the patient was not originally included in the study) and the conventional procedure failed. Later, the patient underwent a robot-assisted procedure (patient 4) and was subsequently included in the study.

\section{Early Follow-Up}

None of the patients had injury due to the trajectory via globe injury, damage to the tooth buds, or a loss of purchase during the active distraction phase. The mean distraction achieved was $23 \mathrm{~mm}$ (range 18-30 mm) (Table 2). Three patients had distractor-related complications in the distraction phase. Two patients required revision surgery for an incomplete osteotomy due to inadequate movement during the distraction. One patient who had an inadequate distraction was lost to follow-up after declining further treatment.
As defined for the purposes of this study, functional improvement was noted at the early follow-up in all 18 patients. According to our PROM-based evaluation, 10 patients $(56 \%)$ had an excellent outcome and 6 patients $(33 \%)$ had a satisfactory outcome (Fig. 6, and Supplemental Figs. 1 and 2). One child who had an inadequate distraction was lost to follow-up (Table 2). In all cases, the degree of OSA had significantly reduced after surgery, and the respiratory conditions had markedly improved.

None of the patients had interim procedures, such as a tracheostomy, for airway relief. One patient with severe airway issues had a postoperative tracheostomy and was weaned off it early, once the distraction was achieved.

Eye closure improved in all patients, and complete closure was seen in 11 patients (11/16) (Table 2). Of the 3 patients who had globe subluxation, 1 required additional tarsorrhaphy. This may have been due to an inadequate distraction or a hypoplastic and deformed (flat compared with normal cupping) orbital floor still unable to retain the globe, in addition to an inadequate distraction. One patient who had tarsorrhaphy at presentation (1/3) experienced a repeated giving away of the sutures of the tarsorrhaphy, leading to persistent subluxation. This led us to occlude his eye entirely and perform an early RAMD.

The functional gain remained in 14 of 16 patients (88\%) 

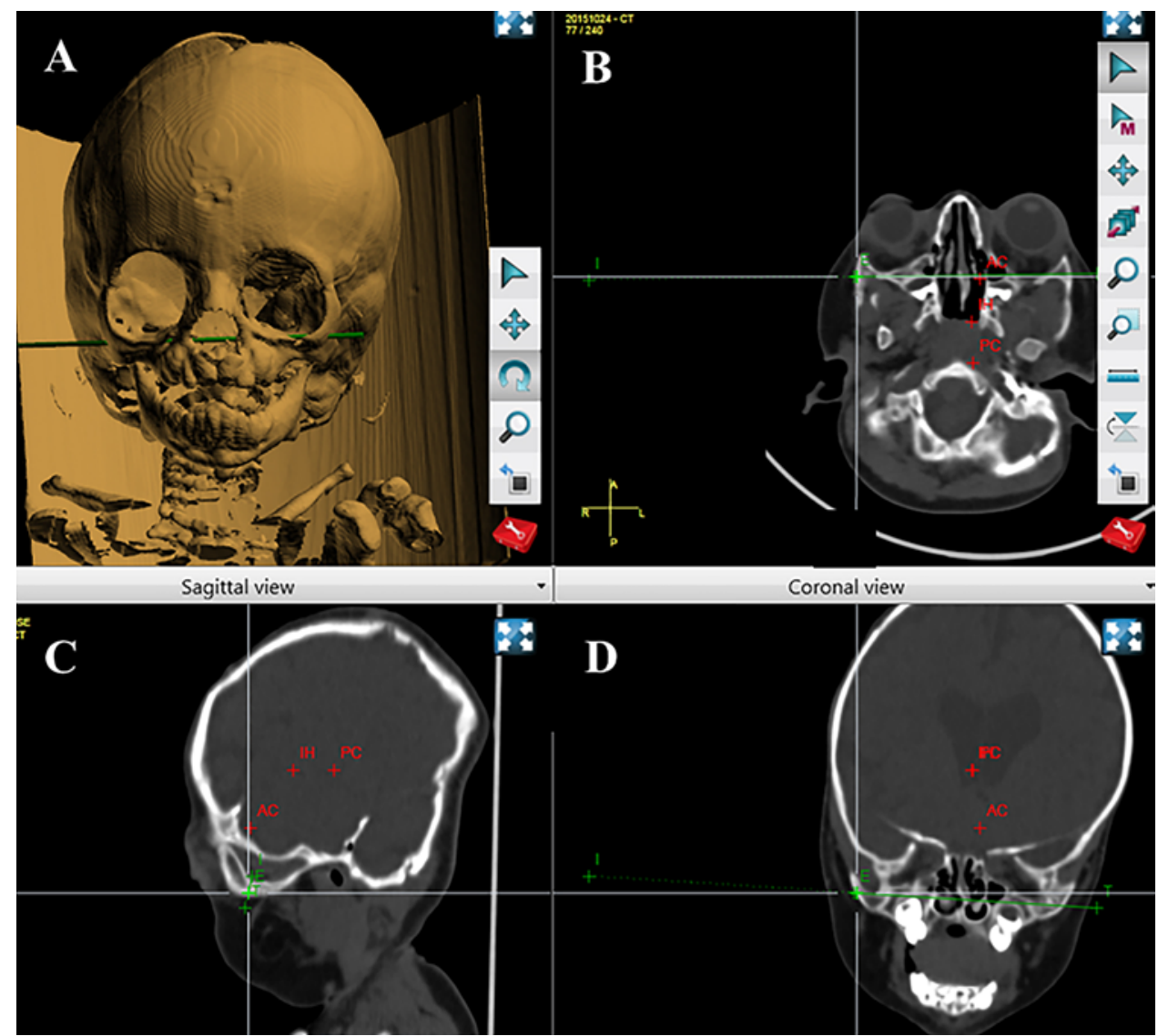

FIG. 3. Planning of robot-assisted transfacial pin insertion on the ROSA planning station. The anterior commissure (AC)-posterior commissure (PC) line, interhemispheric fissure $(\mathrm{IH})$, and the intercommissural basal brain line (all in red) are used as a reference for the midsagittal plane. A and B: A trajectory (green line) for the transfacial pin, with good purchase on the body of the zygoma bones bilaterally, avoiding the maxillary tooth buds and the orbital floor, is planned. $\mathbf{C}$ and D: Good purchase through the zygoma is ensured (green cross). $\mathrm{E}=$ entry; $\mathrm{T}=$ target.

at the last follow-up visit (1 patient had been lost to followup and 1 patient had died). The follow-up period was a mean of 36 months (range 6-72 months). Over the followup period, the distraction results remained without significant relapse.

\section{Discussion}

Craniosynostoses are characterized by the premature closure of one or more of the sutures of the skull. They can occur sporadically or be a part of a syndrome like Apert's, Crouzon, and Pfeiffer. These faciocraniosynostoses have a high incidence of OSA secondary to midface hypoplasia and decreased pharyngeal airway space in all three dimensions (sagittal, vertical, and transversal). Additionally, this may result in exorbitism, globe subluxation, malocclusion, and aesthetic and/or psychosocial problems. ${ }^{3,30} \mathrm{Up}$ to $40 \%$ of patients may require some intervention for OSA. ${ }^{23}$ Craniofacial anomaly is the second most common reason for infant tracheostomy, and the incidence of tracheostomy in children with craniofacial anomalies is approximately 20\%. ${ }^{31,32}$ Adenotonsillectomy may confer some benefit, as even a slight increase in the pharyngeal airway diameter can significantly alter airflow dynamics, increasing the critical closing pressure and reducing OSA severity. However, residual OSA after adenotonsillectomy is common, and patients may require further airway intervention, including insertion of the nasopharyngeal airway, ventilatory support, or surgical intervention such as tracheostomy or midface advancement surgery ${ }^{33}$ Functional indications such as moderate to severe respiratory problems and incomplete eye closure with a risk of corneal damage (severe exorbitism) may require midface surgery at a very young age. Equally, a significant and often underestimated concern is the effect of OSA on the elevation of intraocular pressure and ICP, ${ }^{6-14,34}$ especially in the very young in their crucial stage of development.

Monobloc osteotomy and distraction osteogenesis have become the strategies of choice in many centers to achieve the functional correction of upper airway obstruction, ocular crisis, and the effects of sleep disturbance in very young children, as well as appearance changes at a later age. $3,22,25,26,35-38$

Midface surgery in the first 2 years of life is technically more difficult due to the lower ossification of the facial bones and, irrespective of the technique, has a high failure rate. $22,37,39,40$ Adding to the challenge is that the degree of 

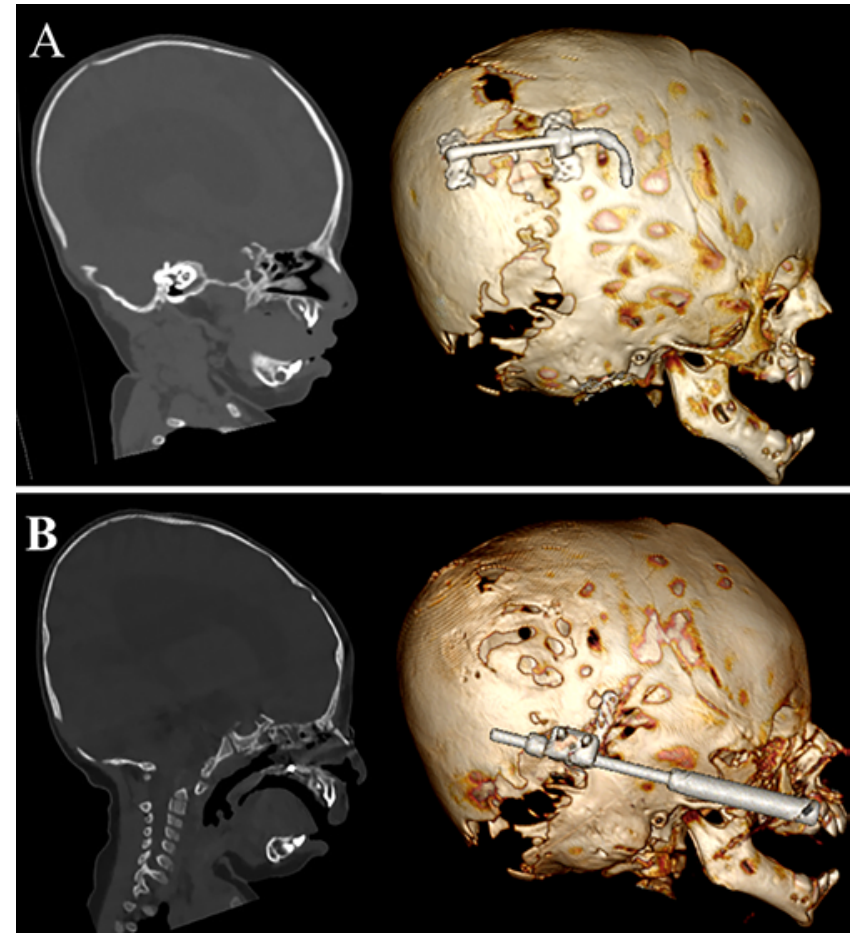

FIG. 4. A: Preoperative sagittal MDCT scan (left) showing a highly compromised airway. A 3D reconstructed image (right) showing the posterior calvarial distractor in situ. These were removed when the patient underwent midface distraction. B: Postoperative sagittal MDCT scan (left) showing improvement of the airway. A 3D reconstructed image (right) showing midface distractor in situ.

advancement could be limited and recurrent midface hypoplasia can occur due to the lack of intrinsic growth of the midface. ${ }^{3,41}$ Various studies of midface advancements, either LeFort III or monobloc, have frequently shown that craniofacial growth after the surgery is interrupted. ${ }^{42,43}$ There is no maxillary growth after an early monobloc, so a second monobloc (or LeFort III) operation after 18 years of age may become necessary. ${ }^{44,45}$ Previous operations increase potential postoperative complications such as oste- itis, which results in partial resorption and complete loss of frontal bone. ${ }^{41}$ For these reasons, unless complex indications like severe airway and ocular crisis occur, postponement until around 7 to 8 years of age has often been proposed. ${ }^{3,39}$ Controversy exists among those who would wait until after 5 years of age and those who prefer to operate as early as possible. ICP and cognitive and psychological long-term follow-up data have demonstrated statistically significant improvement after early surgery. ${ }^{46-49}$ The effect of midface hypoplasia associated with faciocraniosynostosis on the brain is underestimated, as the effect on ICP is difficult to gauge and correlate. Chronic sleep apnea, either central or obstructive, leads to impaired cognitive function and should be explored in the preoperative workup. For all these reasons, Marchac and Arnaud recommended midface distraction around 18 months of age..$^{37,43,44}$

\section{Rationale for the Choice of Technique and Utility of Robotics}

Robotics has been used in neurosurgery for a decade or so. ${ }^{50-69}$ At present, it is used primarily as an assisting and accuracy tool and mainly for placing deep-seated brain electrodes, placing a burr hole in the skull, or placing pedicle screws in the spine. There is no dedicated literature on the application of robotic assistance in craniofacial literature other than our previous work on the broad spectrum of robotic applications in cranial neurosurgery (with 9 patients in the cohort). ${ }^{28}$

Our application of robotics is an instance of a passive system, whereby surgical tools are controlled by moving a cutting guide block or a drilling guide sleeve, while a surgeon handles the tool with free hands (Fig. 2). Unlike optical navigation, theoretically, the application has the additional capability of reducing human error through increased precision in execution, indefatigability, motion scaling, and tremor filtration via mechanical actuation. ${ }^{61}$

The transfacial pin technique has not been widely used $^{70-72}$ Unlike other procedures, it minimizes blood loss and operative times while preserving accuracy in vector selection, in addition to eliminating the risk of communication between nasal and paranasal compartments with the intracranial space. The technique is easy and fast, and
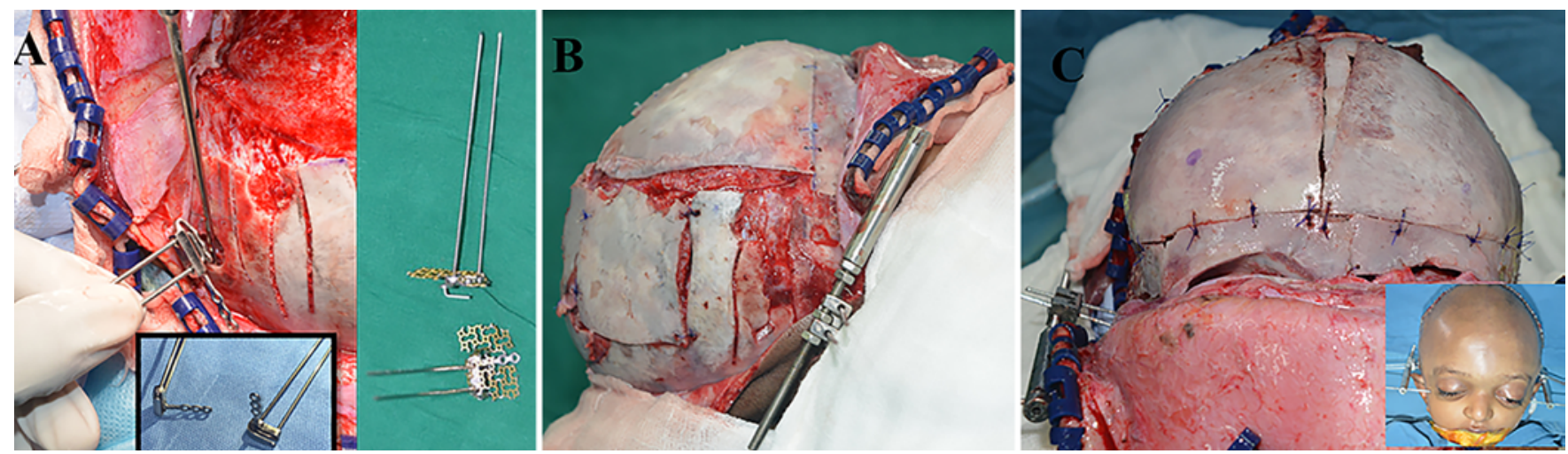

FIG. 5. Intraoperative photographs. A and B: The distractor in situ. The U-shaped arm of the proximal footplate of the distractor $(A)$ is hooked on the temporal bone, and the plate extension is fixed with titanium screws (inset). Lateral profile showing the distractor in situ (B). C: Simultaneous FOAR, and the final appearance of the patient (inset). 
TABLE 2. Assessment of technique and early functional outcome

\begin{tabular}{|c|c|c|c|c|c|c|c|c|c|c|}
\hline \multirow[b]{2}{*}{$\begin{array}{l}\text { Pt } \\
\text { No. }\end{array}$} & \multicolumn{3}{|c|}{ Robotic Transfacial Pin Insertion } & \multicolumn{7}{|c|}{ Midface Distraction Osteogenesis } \\
\hline & $\begin{array}{l}\text { Single-Pass } \\
\text { Pin Insertion }\end{array}$ & $\begin{array}{l}\text { Pin } \\
\text { Parallelism/ } \\
\text { Vector }\end{array}$ & $\begin{array}{c}\text { Successful } \\
\text { Completion w/o } \\
\text { Pin Complications }\end{array}$ & $\begin{array}{l}\text { Quantum of } \\
\text { Distraction } \\
\quad(\mathrm{mm})\end{array}$ & $\begin{array}{c}\text { Airway } \\
\text { (at 6-mo FU) }\end{array}$ & $\begin{array}{c}\text { Eye } \\
\text { Closure }\end{array}$ & Sleep & $\begin{array}{l}\text { Functional } \\
\text { Status at } \\
\text { Last FU }\end{array}$ & PROM & $\begin{array}{c}\text { FU } \\
\text { (mos) }\end{array}$ \\
\hline 1 & Yes & $\begin{array}{l}\text { Acceptable/ } \\
\text { satisfactory }\end{array}$ & Yes & 30 & Improved & Complete & Improved & No issues & Satisfactory & 60 \\
\hline 2 & Yes & $\begin{array}{l}\text { Acceptable/ } \\
\text { satisfactory }\end{array}$ & Yes & NA & $\begin{array}{l}\text { Inadequate } \\
\text { improvement }\end{array}$ & NA & NA & NA & NA & $\begin{array}{l}\text { Lost } \\
\text { to FU }\end{array}$ \\
\hline 3 & Yes & $\begin{array}{l}\text { Acceptable/ } \\
\text { satisfactory }\end{array}$ & Yes & 23 & Resolved & Complete & Improved & No issues & Excellent & 48 \\
\hline 4 & Yes & $\begin{array}{l}\text { Acceptable/ } \\
\text { satisfactory }\end{array}$ & Yes & 26 & Resolved & Partial, improved & Improved & No issues & Satisfactory & 62 \\
\hline 5 & Yes & $\begin{array}{l}\text { Acceptable/ } \\
\text { satisfactory }\end{array}$ & Yes & 21 & Resolved & Complete & Improved & No issues & Excellent & 72 \\
\hline 6 & Yes & $\begin{array}{l}\text { Acceptable/ } \\
\text { satisfactory }\end{array}$ & Yes & 21 & Resolved & Complete & Improved & No issues & Excellent & 55 \\
\hline 7 & Yes & $\begin{array}{l}\text { Acceptable/ } \\
\text { satisfactory }\end{array}$ & Yes & 25 & Improved & Partial, improved & Improved & Relapse & Satisfactory & 50 \\
\hline 8 & Yes & $\begin{array}{l}\text { Acceptable/ } \\
\text { satisfactory }\end{array}$ & Yes & 20 & Improved & Partial, improved & Improved & No issues & Satisfactory & 80 \\
\hline 9 & Yes & $\begin{array}{l}\text { Acceptable/ } \\
\text { satisfactory }\end{array}$ & Yes & 25 & Improved & $\begin{array}{l}\text { Partial, improved; } \\
\text { required tarsorraphy }\end{array}$ & Improved & No issues & Satisfactory & 90 \\
\hline 10 & Yes & $\begin{array}{l}\text { Acceptable/ } \\
\text { satisfactory }\end{array}$ & Yes & 30 & Improved & Partial, improved & Improved & $\begin{array}{l}\text { Relapse of } \\
\text { airway issues }\end{array}$ & Satisfactory & 35 \\
\hline 11 & Yes & $\begin{array}{l}\text { Acceptable/ } \\
\text { satisfactory }\end{array}$ & Yes & 25 & Resolved & Complete & Improved & No Issues & Excellent & 60 \\
\hline 12 & Yes & $\begin{array}{l}\text { Acceptable/ } \\
\text { satisfactory }\end{array}$ & Yes & NA & Improved & Partial, improved & Improved & NA & NA & $\begin{array}{c}\mathrm{Pt} \\
\text { died }\end{array}$ \\
\hline 13 & Yes & $\begin{array}{l}\text { Acceptable/ } \\
\text { satisfactory }\end{array}$ & Yes & 25 & Resolved & Complete & Improved & No issues & Excellent & 36 \\
\hline 14 & Yes & $\begin{array}{l}\text { Acceptable/ } \\
\text { satisfactory }\end{array}$ & Yes & 18 & Resolved & Complete & Improved & No issues & Excellent & 24 \\
\hline 15 & Yes & Skewed & Yes & 23 & Resolved & Complete & Improved & No issues & Excellent & 30 \\
\hline 16 & Yes & $\begin{array}{l}\text { Acceptable/ } \\
\text { satisfactory }\end{array}$ & Yes & 20 & Resolved & Complete & Improved & No issues & Excellent & 12 \\
\hline 17 & Yes & $\begin{array}{l}\text { Acceptable/ } \\
\text { satisfactory }\end{array}$ & Yes & 23 & Resolved & Complete & Improved & No issues & Excellent & 6 \\
\hline 18 & Yes & Skewed & Yes & 18 & Resolved & Complete & Improved & No issues & Excellent & 6 \\
\hline
\end{tabular}

NA = not applicable.

employs very basic, external hardware with low complication rates. ${ }^{71}$

Using transfacial pins for midface distraction in very young patients with midface retrusion and distortion is challenging with a high morbidity rate due to iatrogenic orbital and tooth bud injury $27,70,72$ and technical failure, due to their deformed skull and hypoplastic midface. Lack of maxillary growth and an abnormal remodeling pattern results in small-sized bone structure and disorganized architecture in syndromic craniosynostosis (e.g., small zygoma and maxilla, and a high, arched palate). ${ }^{73}$ The age profile of our patients with midface distraction is much younger than that reported in other series. ${ }^{22,36-39,70}$ Compared with traditional osteotomy techniques, the technique of using transfacial pins ${ }^{27,70}$ allowed us to ad- dress midface distraction considerably early, when the maxillary-zygomatic junction is relatively fragile, and with reduced blood loss. ${ }^{70}$

Although previous authors have used either a freehand technique ${ }^{70}$ or navigation to perform the same, ${ }^{71}$ robotic assistance allowed the safe passage of the pin in a predetermined trajectory with respect to the globe and tooth buds (Fig. 3). Another downside of the transfacial pinning technique is a lack of vector control due to the use of a single anterior facial pin; hence, rotational deformities cannot be controlled. Modification by incorporating a hook to engage the calvaria to prevent posterior displacement of the proximal footplate during distraction and a plate fixed with a screw to prevent dislodgment of the device, along with robotic assistance, allowed us to have reasonable con- 
trol over the vector of the device and parallelism of the transfacial pin.

There is no literature describing similar applications of robotics in craniofacial surgery. In another instance, robot-guided pedicle screw placement offered benefits over conventional dorsal instrumentation techniques, including improved accuracy and safety in pedicle screw insertion. ${ }^{60}$ Our use of the robot as an adjunct for the procedure improved the feasibility, safety, and outcomes, and reduced complications, compared with the previous series on early surgery for midface hypoplasia..$^{22,36-40}$ The challenges of robotic assistance include radiation exposure, trajectory failure, equipment and software failure, failed registration, logistics, time-consuming processes, and high cost.

RAMD and RAFFA have been incorporated very early in our management algorithm for syndromic craniosynostosis, adding a definitive management advantage over interim procedures like tracheostomy with improved procedural safety and minimal invasiveness. There are no studies comparing the long-term results of simultaneous FOAR with midface distraction versus the two-stage procedure of FOAR and subsequent LeFort III with distraction. Our series demonstrates functional gain in terms of quality of life and cognitive improvement. The significant functional gain seen in our cohort of children with craniofacial dysostosis must be taken as an important indicator from our work, although it would be difficult to quantify gains in these spheres of child development. We emphatically recommend early midface advancement in at least those patients with severe symptomatic involvement of the midface, along with addressing raised ICP. However, the need for reoperation and secondary procedures at a later age for aesthetic and functional needs may be very high.

\section{Limitations}

The most critical limitation of our study is that the longitudinal benefit of our updated algorithm has not been evaluated. The long-term outcomes in patients in whom we performed early midface distraction remain unknown, as the patients have not yet reached skeletal maturity. The aesthetic outcome may also be suboptimal, but functional outcome was the critical factor for our decision to proceed with early midface distraction in our cohort; any aesthetic benefit to these children, as a result of modifying the retruded midface, is an additional gain.

Additionally, the PROM we used to evaluate the results of early midface distraction has not been validated. Although it lacks validation, there is a clear expression of improvement.

\section{Envisioning the Role for Robotics in Craniofacial Surgery}

Robotics is expanding its utility in many fields of surgery, and our study is but a small foray of robotics into the field of craniofacial surgery. We envision future applications of robotics, augmented reality, and artificial intelligence in preoperative planning and customized surgical execution. Together, these technologies may reduce surgical time and morbidity, and improve outcome predictability, the biggest challenges faced in these surgeries.
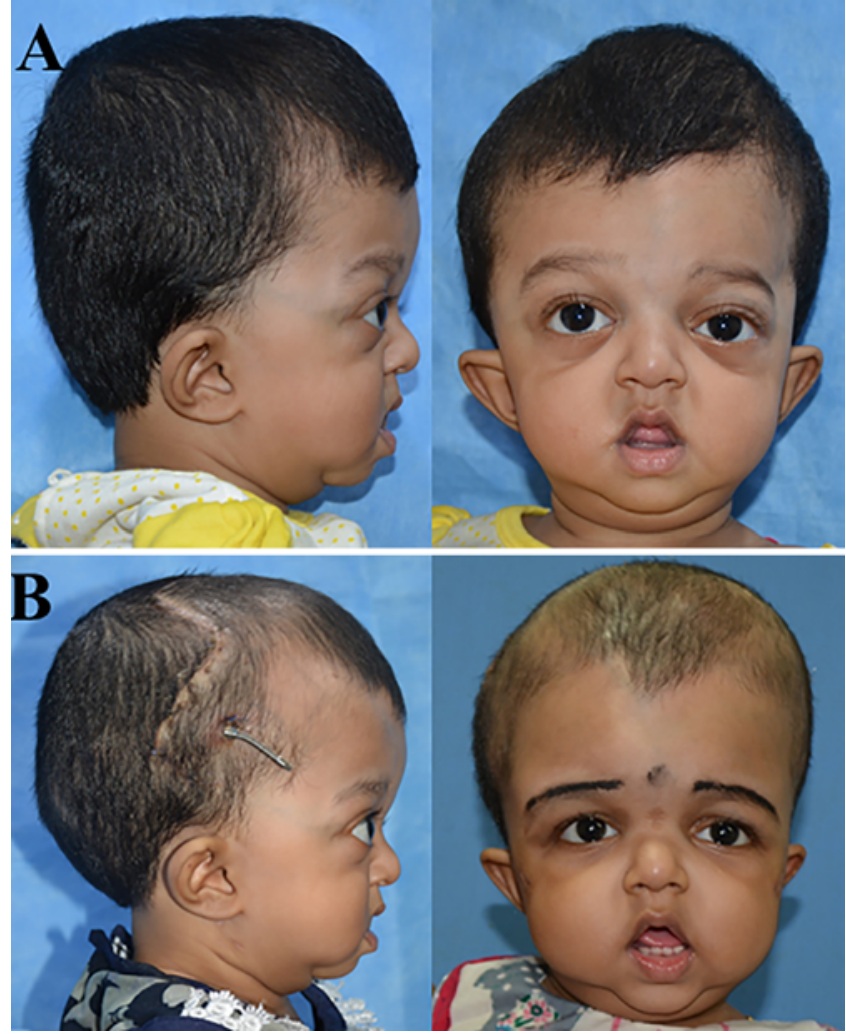

FIG. 6. Patient 17. A: A 15-month-old child presented with hydrocephalus and signs of raised ICP. The patient had posterior calvarial vault distraction (PCVD) at 7 months of age. Despite PCVD, the fontanelle remained on the fuller side. Additionally, the child had severe airway issues with recurrent episodes of URTI. B: Subsequently, she underwent RAFFA (during the removal of posterior calvarial distractors). On follow-up, the patient continued to have macrocephaly with hydrocephalus and subsequently underwent ventriculoperitoneal shunt placement for hydrocephalus.

\section{Conclusions}

Our early RAFFA and RAMD protocols for craniofacial dysostoses give a significant functional advantage in the very young with severe airway and globe issues. We have demonstrated the utility and safety of robotic assistance to perform transfacial pin insertion for RAFFA or RAMD.

\section{Acknowledgments}

We wish to sincerely acknowledge Mr. Kishore Kumar of Schiller India for his assistance in retrieving screenshots used in the paper.

\section{References}

1. Tonello C, Cevidanes LHS, Ruellas ACO, Alonso N. Midface morphology and growth in syndromic craniosynostosis patients following frontofacial monobloc distraction. $\mathrm{J} \mathrm{Cra-}$ niofac Surg. 2021;32(1):87-91.

2. Raposo-Amaral CE, Vieira PH, Denadai R, Ghizoni E, Raposo-Amaral CA. Treating syndromic craniosynostosis with monobloc facial bipartition and internal distractor devices: destigmatizing the syndromic face. Clin Plast Surg. 2021;48(3):521-529. 
3. Mathijssen IMJ. Updated guideline on treatment and management of craniosynostosis. J Craniofac Surg. 2021;32(1): 371-450.

4. Raposo-Amaral CE, Denadai R, Oliveira YM, Ghizoni E, Raposo-Amaral CA. Apert syndrome management: changing treatment algorithm. J Craniofac Surg. 2020;31(3):648-652.

5. Renier D, Lajeunie E, Arnaud E, Marchac D. Management of craniosynostoses. Childs Nerv Syst. 2000;16(10-11):645-658.

6. de Jong T, Bannink N, Bredero-Boelhouwer HH, et al. Longterm functional outcome in 167 patients with syndromic craniosynostosis; defining a syndrome-specific risk profile. $J$ Plast Reconstr Aesthet Surg. 2010;63(10):1635-1641.

7. Gonsalez SL, Thompson D, Hayward R, Lane R. Breathing patterns in children with craniofacial dysostosis and hindbrain herniation. Eur Respir J. 1998;11(4):866-872.

8. Doyle KJ, Tami TA. Increased intracranial pressure and blindness associated with obstructive sleep apnea. Otolaryngol Head Neck Surg. 1991;105(4):613-616.

9. Jennum P, Børgesen SE. Intracranial pressure and obstructive sleep apnea. Chest. 1989;95(2):279-283.

10. Lee AG, Golnik K, Kardon R, Wall M, Eggenberger E, Yedavally S. Sleep apnea and intracranial hypertension in men. Ophthalmology. 2002;109(3):482-485.

11. Loeppky JA, Miranda FG, Eldridge MW. Abnormal cerebrovascular responses to $\mathrm{CO} 2$ in sleep apnea patients. Sleep. 1984;7(2):97-109.

12. Pasterkamp H, Cardoso ER, Booth FA. Obstructive sleep apnea leading to increased intracranial pressure in a patient with hydrocephalus and syringomyelia. Chest. 1989;95(5): 1064-1067.

13. Purvin VA, Kawasaki A, Yee RD. Papilledema and obstructive sleep apnea syndrome. Arch Ophthalmol. 2000;118(12): 1626-1630.

14. Wall M, Purvin V. Idiopathic intracranial hypertension in men and the relationship to sleep apnea. Neurology. 2009; 72(4):300-301.

15. Zhang B, Zhu DM, Zhao W, et al. Selective microstructural integrity impairments of the anterior corpus callosum are associated with cognitive deficits in obstructive sleep apnea. Brain Behav. 2019;9(12):e01482.

16. Beebe DW, Gozal D. Obstructive sleep apnea and the prefrontal cortex: towards a comprehensive model linking nocturnal upper airway obstruction to daytime cognitive and behavioral deficits. J Sleep Res. 2002;11(1):1-16.

17. Krysta K, Bratek A, Zawada K, Stepańczak R. Cognitive deficits in adults with obstructive sleep apnea compared to children and adolescents. J Neural Transm (Vienna). 2017; 124(1)(suppl 1):187-201.

18. Macchitella L, Romano DL, Marinelli CV, et al. Neuropsychological and socio-cognitive deficits in patients with obstructive sleep apnea. J Clin Exp Neuropsychol. 2021;43(5): 514-533.

19. Nair D, Ramesh V, Gozal D. Cognitive deficits are attenuated in neuroglobin overexpressing mice exposed to a model of obstructive sleep apnea. Front Neurol. 2018;9:426.

20. Olaithe M, Bucks RS, Hillman DR, Eastwood PR. Cognitive deficits in obstructive sleep apnea: insights from a metareview and comparison with deficits observed in COPD, insomnia, and sleep deprivation. Sleep Med Rev. 2018;38: 39-49.

21. Pan YY, Deng Y, Xu X, Liu YP, Liu HG. Effects of continuous positive airway pressure on cognitive deficits in middleaged patients with obstructive sleep apnea syndrome: a metaanalysis of randomized controlled trials. Chin Med J (Engl). 2015;128(17):2365-2373

22. Ahmad F, Cobb ARM, Mills C, Jones BM, Hayward RD, Dunaway DJ. Frontofacial monobloc distraction in the very young: a review of 12 consecutive cases. Plast Reconstr Surg. 2012;129(3):488e-497e.
23. Driessen C, Joosten KF, Bannink N, et al. How does obstructive sleep apnoea evolve in syndromic craniosynostosis? A prospective cohort study. Arch Dis Child. 2013;98(7):538-543.

24. Way BLM, Khonsari RH, Karunakaran T, et al. Correcting exorbitism by monobloc frontofacial advancement in Crouzon-Pfeiffer syndrome: an age-specific, time-related, controlled study. Plast Reconstr Surg. 2019;143(1):121e-132e.

25. McCarthy JG, Grayson B, Bookstein F, Vickery C, Zide B. Le Fort III advancement osteotomy in the growing child. Plast Reconstr Surg. 1984;74(3):343-354.

26. Meazzini MC, Mazzoleni F, Caronni E, Bozzetti A. Le Fort III advancement osteotomy in the growing child affected by Crouzon's and Apert's syndromes: presurgical and postsurgical growth. J Craniofac Surg. 2005;16(3):369-377.

27. Coeugniet E, Pellerin P, Wolber A, Dhellemmes P, Vinchon $M$. Fifteen years of experience with the midfacial distraction without maxillary osteotomy protocol. Childs Nerv Syst. 2014;30(4):681-688.

28. Pillai A, Ratnathankom A, Ramachandran SN, Udayakumaran S, Subhash P, Krishnadas A. Expanding the spectrum of robotic assistance in cranial neurosurgery. Oper Neurosurg (Hagerstown). 2019;17(2):164-173.

29. Udayakumaran S, Onyia CU. Customizable rigid head fixation for infants: technical note. Childs Nerv Syst. 2016;32(1): 159-161.

30. Mathijssen I, de Goederen R, Versnel SL, Joosten KFM, van Veelen MC, Tasker RC. Letter to the Editor. Raised intracranial pressure and cognitive delay in craniosynostosis. $J$ Neurosurg Pediatr. 2017;20(5):498-502.

31. Perkins JA, Sie KC, Milczuk H, Richardson MA. Airway management in children with craniofacial anomalies. Cleft Palate Craniofac J. 1997;34(2):135-140.

32. Sculerati N, Gottlieb MD, Zimbler MS, Chibbaro PD, McCarthy JG. Airway management in children with major craniofacial anomalies. Laryngoscope. 1998;108(12):1806-1812.

33. Tan HL, Kheirandish-Gozal L, Abel F, Gozal D. Craniofacial syndromes and sleep-related breathing disorders. Sleep Med Rev. 2016;27:74-88.

34. Quinn AG, Gouws P, Headland S, et al. Obstructive sleep apnea syndrome with bilateral papilledema and vision loss in a 3-year-old child. J AAPOS. 2008;12(2):197-199.

35. McCarthy JG, Epstein F, Sadove M, Grayson B, Zide B. Early surgery for craniofacial synostosis: an 8-year experience. Plast Reconstr Surg. 1984;73(4):521-533.

36. Witherow H, Dunaway D, Evans R, et al. Functional outcomes in monobloc advancement by distraction using the rigid external distractor device. Plast Reconstr Surg. 2008; 121(4):1311-1322.

37. Arnaud E, Di Rocco F. Faciocraniosynostosis: monobloc frontofacial osteotomy replacing the two-stage strategy? Childs Nerv Syst. 2012;28(9):1557-1564.

38. Caterson EJ, Shetye PR, Grayson BH, McCarthy JG. Surgical management of patients with a history of early Le Fort III advancement after they have attained skeletal maturity. Plast Reconstr Surg. 2013;132(4):592e-601e.

39. Gwanmesia I, Jeelani O, Hayward R, Dunaway D. Frontofacial advancement by distraction osteogenesis: a long-term review. Plast Reconstr Surg. 2015;135(2):553-560.

40. Patel N, Fearon JA. Treatment of the syndromic midface: a long-term assessment at skeletal maturity. Plast Reconstr Surg. 2015;135(4):731e-742e.

41. Arnaud E, Marchac D, Renier D. Reduction of morbidity of the frontofacial monobloc advancement in children by the use of internal distraction. Plast Reconstr Surg. 2007;120(4): 1009-1026.

42. Shetye PR, Davidson EH, Sorkin M, Grayson BH, McCarthy JG. Evaluation of three surgical techniques for advancement of the midface in growing children with syndromic craniosynostosis. Plast Reconstr Surg. 2010;126(3):982-994. 
43. Marchac D, Arnaud E. Midface surgery from Tessier to distraction. Childs Nerv Syst. 1999;15(11-12):681-694.

44. Mathijssen IM. Guideline for care of patients with the diagnoses of craniosynostosis: working group on craniosynostosis. J Craniofac Surg. 2015;26(6):1735-1807.

45. Cruz AA, Akaishi PM, Arnaud E, Marchac D, Renier D. Palpebral fissure changes after monobloc frontofacial advancement in faciocraniosynostosis. J Craniofac Surg. 2008;19(1): 106-109.

46. Gonsalez S, Hayward R, Jones B, Lane R. Upper airway obstruction and raised intracranial pressure in children with craniosynostosis. Eur Respir J. 1997;10(2):367-375.

47. Alkan U, Nachalon Y, Weiss P, et al. Effects of surgery for obstructive sleep apnea on cognitive function and driving performance. Sleep Breath. 2021;25(3):1593-1600.

48. Poca MA, Ferré A, de la Calzada MD, Moncho D, Fernandez-Torrelles S, Sahuquillo J. $\mathrm{CO}_{2}$-induced intracranial hypertension and high-amplitude B-waves in a patient with Chiari 1 malformation and sleep apnea syndrome that resolved following CPAP therapy. Acta Neurochir (Wien). 2021;163(11):3075-3082.

49. Beqiri E, Czosnyka M, Lalou AD, et al. Influence of mildmoderate hypocapnia on intracranial pressure slow waves activity in TBI. Acta Neurochir (Wien). 2020;162(2):345-356.

50. Benabid AL, Hoffmann D, Ashraf A, Koudsie A, Esteve F, Le Bas JF. Robotics in neurosurgery: current status and future prospects. Article in French. Chirurgie. 1998;123(1): 25-31.

51. Benabid AL, Hoffmann D, Seigneuret E, Chabardes S. Robotics in neurosurgery: which tools for what? Acta Neurochir Suppl. 2006;98:43-50.

52. Cardinale F. Commentary: The path to surgical robotics in neurosurgery. Oper Neurosurg (Hagerstown). 2021;21(2): E167-E168.

53. Doulgeris JJ, Gonzalez-Blohm SA, Filis AK, Shea TM, Aghayev K, Vrionis FD. Robotics in neurosurgery: evolution, current challenges, and compromises. Cancer Contr. 2015; 22(3):352-359.

54. Giorgi C, Sala R, Riva D, Cossu A, Eisenberg H. Robotics in child neurosurgery. Childs Nerv Syst. 2000;16(10-11):832834.

55. Khanna O, Beasley R, Franco D, DiMaio S. The path to surgical robotics in neurosurgery. Oper Neurosurg (Hagerstown). 2021;20(6):514-520.

56. Lafuente J. Robotics assistance in neurosurgery-improving the outcome for our patients. Acta Neurochir (Wien). 2018; 160(10): 1889 .

57. Madhavan K, Kolcun JPG, Chieng LO, Wang MY. Augmented-reality integrated robotics in neurosurgery: are we there yet? Neurosurg Focus. 2017;42(5):E3.

58. McBeth PB, Louw DF, Rizun PR, Sutherland GR. Robotics in neurosurgery. Am J Surg. 2004;188(4A Suppl):68S-75S.

59. Menaker SA, Shah SS, Snelling BM, Sur S, Starke RM, Peterson EC. Current applications and future perspectives of robotics in cerebrovascular and endovascular neurosurgery. $J$ Neurointerv Surg. 2018;10(1):78-82.

60. Naros G, Machetanz K, Grimm F, Roser F, Gharabaghi A, Tatagiba M. Framed and non-framed robotics in neurosurgery: a 10-year single-center experience. Int J Med Robot. 2021;17(5):e2282.

61. Nathoo N, Cavuşoğlu MC, Vogelbaum MA, Barnett GH. In touch with robotics: neurosurgery for the future. Neurosurgery. 2005;56(3):421-433.
62. Nathoo N, Pesek T, Barnett GH. Robotics and neurosurgery. Surg Clin North Am. 2003;83(6):1339-1350.

63. Pandya S, Motkoski JW, Serrano-Almeida C, Greer AD, Latour I, Sutherland GR. Advancing neurosurgery with image-guided robotics. J Neurosurg. 2009;111(6):1141-1149.

64. Sekhar LN, Tariq F, Kim LJ, Pridgeon J, Hannaford B. Commentary: Virtual reality and robotics in neurosurgery. Neurosurgery. 2013;72(suppl 1):1-6.

65. Sutherland G. Introduction to virtual reality and robotics in neurosurgery. Neurosurgery. 2013;72(suppl 1):7.

66. Wang MY, Goto T, Tessitore E, Veeravagu A. Introduction. Robotics in neurosurgery. Neurosurg Focus. 2017;42(5):E1.

67. Wu C, Wagner CR. Introduction: Surgical Robotics in Neurosurgery Review Series. Oper Neurosurg (Hagerstown). 2021; 20(6):513.

68. Young RF. Application of robotics to stereotactic neurosurgery. Neurol Res. 1987;9(2):123-128.

69. Zamorano L, Li Q, Jain S, Kaur G. Robotics in neurosurgery: state of the art and future technological challenges. Int J Med Robot. 2004;1(1):7-22.

70. Coeugniet E, Dhellemmes P, Vinchon M, Wolber A, Pellerin P. Midfacial distraction without osteotomy using a transfacial pin and external devices. J Craniofac Surg. 2012;23(1):184189.

71. Goldstein JA, Paliga JT, Bailey RL, Heuer GG, Taylor JA. Posterior vault distraction with midface distraction without osteotomy as a first stage for syndromic craniosynostosis. $J$ Craniofac Surg. 2013;24(4):1263-1267.

72. Mitsukawa N, Satoh K. Midfacial distraction using a transfacial pinning technique for syndromic craniosynostosis with obstructive respiratory disorders. J Plast Reconstr Aesthet Surg. 2010;63(12):1990-1994.

73. Kreiborg S, Pruzansky S. Craniofacial growth in premature craniofacial synostosis. Scand J Plast Reconstr Surg. 1981; 15(3):171-186.

\section{Disclosures}

The authors report no conflict of interest concerning the materials or methods used in this study or the findings specified in this paper.

\section{Author Contributions}

Conception and design: Udayakumaran, Subash. Acquisition of data: Udayakumaran, Krishnadas. Analysis and interpretation of data: Udayakumaran. Drafting the article: Udayakumaran, Subash. Critically revising the article: Udayakumaran, Subash. Reviewed submitted version of manuscript: all authors. Approved the final version of the manuscript on behalf of all authors: Udayakumaran. Administrative/technical/material support: Udayakumaran. Study supervision: Udayakumaran.

\section{Supplemental Information \\ Online-Only Content}

Supplemental material is available online.

Supplemental Table and Figures. https://thejns.org/doi/ suppl/10.3171/2021.10.FOCUS21515.

\section{Correspondence}

Suhas Udayakumaran: Amrita Institute of Medical Sciences and Research Centre, Kochi, Kerala, India.dr.suhas@gmail.com. 